\title{
EIGENVALUES OF POSITIVE INTEGRAL OPERATORS WITH LAPLACE TRANSFORM-TYPE KERNELS
}

\author{
YÜKSEL SOYKAN \\ Department of Mathematics, Art and Science Faculty, Zonguldak Karaelmas University, 67100, \\ Zonguldak, Turkey \\ e-mail: yuksel_soykan@hotmail.com \\ and GRAHAM LITTLE ${ }^{1}$ \\ Department of Mathematics, University of Manchester, Manchester, UK
}

(Received 4 January 2009; accepted 5 October 2009)

\begin{abstract}
The aim of this paper is to prove a theorem concerning asymptotic estimates of eigenvalues of certain positive integral operators with Laplace transformtype kernels.

2000 Mathematics Subject Classification. Primary 45C05, 45H05, 45P05; secondary 47B38.
\end{abstract}

1. Introduction. Our work is motivated by a classic paper of H. Widom. In [12] Widom considers integral operators on $L^{2}(-1,1)$ with kernels of the form $K(s, t)=$ $\widehat{\psi}(s-t)$, where $\psi$ is a continuous, positive even function on $\mathbb{R}$, which vanishes at infinity, and $\widehat{\psi}$ is the Fourier transform of $\psi$

$$
\widehat{\psi}(s)=\int_{-\infty}^{\infty} \psi(u) e^{i s u} \quad(-\infty<s<\infty) .
$$

There are three main theorems in [12]. It is Widom's theorem II which is of interest in the present context and the aim of this paper is to prove a theorem, Theorem 4, similar to Widom's Theorem II.

Let $I$ be a closed (bounded) sub-interval of the real line $\mathbb{R}$. To prove our main theorem, Theorem 4, we need to recall some definitions and results of [11]. The first definition we require is the following.

Definition 1. A function $K$ of two complex variables will be called an $A^{+}$kernel on $I \times I$, and we will write $K \in A^{+}(I)$, if

(i) there is an open neighbourhood $G$ of $I$ in $\mathbb{C}$ such that $K$ is continuous on

$$
G \times G \text {. }
$$

(ii) $K(z, w)=\overline{K(w, z)}$, for all $z, w \in G$.

(iii) For each $w \in G$, the function $z \longmapsto K(z, w)$ is analytic on $G$.

${ }^{1}$ Retired 
(iv) The compact symmetric integral operator $T_{K I}$ on $L^{2}(I)$ :

$$
T_{K I} f(s)=\int_{I} K(s, t) f(t) d t \quad\left(f \in L^{2}(I), s \in I\right)
$$

is positive in the sense that $\left(T_{K I} f, f\right) \geq 0$, for all $f \in L^{2}(I)$.

We are interested in finding asymptotic estimates of eigenvalues of explicit kernels in $A^{+}(I)$. In practice, we shall probably be given $K(s, t)$ for real values of $s, t$ at the outset. It will always be possible to extend $K$ to a function of complex variables by replacing $s$ with $z$ and $t$ with $\bar{w}$. Consider, for instance, the simple example

$$
K(s, t)=\frac{1}{s+t}, \quad I=[a, b],
$$

where $0<a<b$. An extension of $K$ to a $A^{+}$kernel on $I$ is given by

$$
K(z, w)=\frac{1}{z+\bar{w}} .
$$

In the usual way, we shall always assume that the eigenvalues of $T_{K I}$ are ordered into a decreasing sequence $\lambda_{0}\left(T_{K I}\right) \geq \lambda_{1}\left(T_{K I}\right) \geq \lambda_{2}\left(T_{K I}\right) \geq \ldots$ tending to 0 , with repetitions to account for multiplicities. We shall write $\lambda_{n}\left(T_{K I}\right)$ and $\lambda_{n}(K, I)$ interchangeably for these eigenvalues. Given two sequences $\left(a_{n}\right)$ and $\left(b_{n}\right)$ of non-negative reals, we shall write $a_{n} \simeq b_{n}$ when $a_{n}=O\left(b_{n}\right)$ and $b=O\left(a_{n}\right)$. It is known [4] that if $K \in A^{+}(I)$, then $\lambda_{n}(K, I)=O\left(r^{n}\right)$, for some $r$ satisfying $0<r<1$, but the estimates of this paper will be of the sharper form $\lambda_{n}(K, I) \simeq n^{c} r^{n}$, where $c \in \mathbb{R}$ and $0<r<1$.

In [5] and [7] Little considered power series kernels, that is kernels of the form $\sum_{n=0}^{\infty} a_{n} s^{n} t^{n}$, where $a_{n} \geq 0$, for all $n$. Our main result will be derived from the following theorem, see Theorem 2 of [7]. The term $q(k)$ will be explained immediately after the statement of the result.

THEOREM 2. Let $K(s, t)=\sum_{n=0}^{\infty} a_{n} s^{n} t^{n}$, where $a_{n}>0$, for all $n$, and $a_{n} \simeq n^{c}$, for some $c \in \mathbb{R}$. If $J=[\alpha, \beta]$, where $-1<\alpha<\beta<1$, then the integral operator $T_{K J}$ on $L^{2}(J)$ :

$$
T_{K J} f(s)=\int_{J} K(s, t) f(t) d t \quad\left(f \in L^{2}(J), s \in J\right)
$$

is compact and positive, and its eigenvalues satisfy

$$
\lambda_{n}(K, J) \simeq n^{c} q\left(\frac{\beta-\alpha}{1-\alpha \beta}\right)^{n} .
$$

Here $q=q(k)$ is Jacobi's nome with modulus $k$ as in the theory of elliptic functions and integrals. It is defined for $0<k<1$ by

$$
q(k)=\exp \left(-\pi K^{\prime} / K\right)
$$

where

$$
K=\int_{0}^{\pi / 2} \frac{d \theta}{\left(1-k^{2} \sin ^{2} \theta\right)^{\frac{1}{2}}}, \quad K^{\prime}=\int_{0}^{\pi / 2} \frac{d \theta}{\left(1-\ell^{2} \sin ^{2} \theta\right)^{\frac{1}{2}}}
$$


and $\ell=\left(1-k^{2}\right)^{\frac{1}{2}}$. This parameter $q(k)$ arises naturally in the theory of Jacobian elliptic functions. A detailed knowledge of the theory of elliptic functions and integrals is not necessary to understand our methods in this paper, though the reader should note that $q$ is a strictly increasing continuous function on the open interval $(0,1)$ and that

$$
\lim _{k \rightarrow 0} q(k)=0, \quad \lim _{k \rightarrow 1} q(k)=1 .
$$

Detailed accounts of elliptic functions and integrals can be found in [3] and [8] and there is good introductory account in [9]. Numerical values of $K, K^{\prime}$ and $q$ are tabulated in handbooks such as [1].

Here we shall study Laplace transform kernels:

$$
K(s, t)=\int_{0}^{\infty} \psi(u) e^{-u(s+t)} d u \quad(s, t>0),
$$

where $\psi$ is a locally integrable, non-negative function on $(0, \infty)$ and satisfies further conditions to be given in Definition 3.

A Laplace transform kernel will be defined using a function $\psi$ on $(0, \infty)$ satisfying conditions analogous to those imposed on the sequence $\left(a_{n}\right)$ as in the Theorem 2 . For non-negative functions $\phi, \psi$ on $(0, \infty)$ we will write $\phi(u) \simeq \psi(u)$ as $u \rightarrow \infty$ when there are constants $M, m>0$ and $u_{0} \geq 0$ such that

$$
m \phi(u) \leq \psi(u) \leq M \phi(u) \quad\left(u \geq u_{0}\right) .
$$

Definition 3. (i) A function $\psi$ on $(0, \infty)$ will said to be of class $P(0, \infty)$ if it is non-negative and locally integrable and if, for all $\delta>0$,

$$
\psi(u)=O\left(e^{\delta u}\right) \text { as } u \rightarrow \infty .
$$

(ii) A function $\psi$ will said to be of class $P_{c}(0, \infty)$, where $c \in \mathbb{R}$, if it is non-negative and locally integrable and

$$
\psi(u) \simeq u^{c} \text { as } u \rightarrow \infty .
$$

(iii) Given $\psi \in P(0, \infty)$ let $K_{\psi}$ denote the function

$$
K_{\psi}(s, t)=\int_{0}^{\infty} \psi(u) e^{-u(s+t)} d u \quad(s, t>0)
$$

(iv) Given $\psi \in P(0, \infty)$ and a closed interval $I=[a, b]$, where $0<a<b<\infty$ let $T_{\psi I}$ denote the integral operator on $L^{2}(I)$ with kernel $K_{\psi}$ :

$$
T_{\psi I} f(s)=\int_{I} K_{\psi}(s, t) f(t) d t \quad\left(f \in L^{2}(I), s \in I\right) .
$$

Clearly, $P_{c}(0, \infty) \subseteq P(0, \infty)$, for all $c \in \mathbb{R}$, and a $\psi \in P(0, \infty)$ belongs to at most one $P_{c}(0, \infty)$. If $\psi \in P(0, \infty)$, then its Laplace transform is analytic on the open right half-plane $G$, and if $I$ is as in (iv), then $K_{\psi}$ is continuous and real-symmetric on $I \times I$, so that $T_{\psi I}$ is a compact symmetric operator on $L^{2}(I)$; moreover, $T_{\psi I}$ is positive. 
We can now state our main result.

TheOREM 4. Let $\psi \in P_{c}(0, \infty)$, where $c \in \mathbb{R}$ and let $I=[a, b]$, where $0<a<b<$ $\infty$. If

$$
K_{\psi}(s, t)=\int_{0}^{\infty} \psi(u) e^{-u(s+t)} d u \quad(s, t>0)
$$

and if $T_{\psi I}$ is the integral operator on $L^{2}(I)$ with kernel $K_{\psi}$,

$$
T_{\psi I} f(s)=\int_{I} K_{\psi}(s, t) f(t) d t \quad\left(f \in L^{2}(I), s \in I\right),
$$

then $T_{\psi I}$ is compact and positive and its eigenvalues satisfy

$$
\lambda_{n}\left(T_{\psi I}\right)=\lambda_{n}\left(K_{\psi}, I\right) \simeq n^{c} q\left(\frac{b-a}{b+a}\right)^{n} .
$$

REMARK 5. The proof of above theorem when $c>-1$ is given in [11]. So we shall prove the theorem for the case $c \leq-1$.

2. Reductions and preliminaries. If $T$ is a compact positive operator on a Hilbert space $H$, then its eigenvalues $\lambda_{0}(T) \geq \lambda_{1}(T) \geq \lambda_{2}(T) \geq \ldots$ can be determined by the Courant-Weyl formula:

$$
\lambda_{n}(T)=\inf _{\operatorname{dim} E^{\perp}=n} \sup _{\substack{f \in E \\\|f\|=1}}\langle T f, f\rangle \quad(n \geq 0)
$$

where $E$ denotes a closed sub-space of $H$ and $E^{\perp}$ is its orthogonal complement.

For the formula, see Section 95 of [10], but note that we are using non-negative rather than positive integers to label our eigenvalues. We shall need the following lemma (for a proof, see Lemma 2.1 of [11]) in the proof of Theorem 4.

Lemma 6. Let $H$ be a Hilbert space and suppose that $A, B$ are compact positive operators on $H$ and that $R$ is a continuous symmetric operator on $H$ of finite rank. Also suppose that $H_{1}$ is a Hilbert space, $S$ is a compact positive operator on $H_{1}$ and $V: H_{1} \rightarrow H$ is a continuous operator.

(i) $\left|\lambda_{n}(A)-\lambda_{n}(B)\right| \leq\|A-B\| \quad(n \geq 0)$.

(ii) $\lambda_{n}\left(V S V^{*}\right) \leq\|V\|^{2} \lambda_{n}(S) \quad(n \geq 0)$.

(iii) If, in addition, $V$ is invertible, then

$$
\lambda_{n}\left(V S V^{*}\right) \simeq \lambda_{n}(S) .
$$

(iv) If $A+R \geq 0$ and rank $R \leq N<\infty$, then

$$
\lambda_{n}(A+R) \leq \lambda_{n-N}(A) \quad(n \geq N) .
$$

From now on, we shall always assume that $I$ and $J$ are (bounded) closed subintervals of $\mathbb{R}$. The term positive definite will be used for kernels, while the term positive will be reserved for operators. Thus the continuous, symmetric kernel $K$ on $I \times I$ will be 
called positive definite if and only if the integral operator $T_{K I}$ on $L^{2}(I)$ is positive, that is $\left\langle T_{K I} f, f\right\rangle \geq 0$, for all $f \in L^{2}(I)$. In this case we will write

$$
K(s, t) \geq^{\circ} 0 \quad(s, t \in I)
$$

and more generally, if $L$ is another positive definite kernel on $I \times I$, we will write

$$
K(s, t) \geq^{\circ} L(s, t) \quad(s, t \in I)
$$

when $T_{K I}-T_{L I} \geq 0$ in the operator sense.

We shall need the following lemma as well.

LeMmA 7. Let $K$, L be continuous positive definite kernels on $I \times I$.

(i) The pointwise product $K L$ is positive definite.

(ii) Suppose that $T_{L I}=S S^{*}$, where $S$ is a compact operator mapping some Hilbert space $H$ into $L^{2}(I)$, suppose that $\rho \in H$ is non-zero and that $\sigma=S \rho$ is continuous, then

$$
K(s, t) L(s, t) \geq^{0} \mu \sigma(s) K(s, t) \overline{\sigma(t)} \quad(s, t \in I),
$$

where $\mu=1 /\|\rho\|^{2}$.

Proof. For a proof, see a corollary of proposition 2 of [6] or Lemma 2.2 of [11].

From now on our notation will be as set out in Definition 3 and Theorem 4. Thus $I=[a, b]$ always satisfies $0<a<b<\infty$, $\psi$ is a non-negative, locally integrable function on $(0, \infty)$, and $K_{\psi}(s, t)$ is the Laplace transform of $\psi$ evaluated at $s+t$.

The following lemma shows that if $\psi \in P_{c}(0, \infty)$, then the asymptotic behaviour of the eigenvalues of $T_{\psi I}$ depends only on $c$. This lemma gives us reductions to proof Theorem 4.

Lemma 8. Let $I=[a, b]$, where $a>0$, and let $c \in \mathbb{R}$. If $\phi, \psi \in P_{c}(0, \infty)$, then

$$
\lambda_{n}\left(K_{\psi}, I\right) \simeq \lambda_{n}\left(K_{\phi}, I\right) .
$$

Proof. For the proof, see Lemma 2.3 of [11].

This lemma shows us that, for a given $c \in \mathbb{R}$, we need only to verify Theorem 4 for one special case $\psi \in P_{c}(0, \infty)$. A good choice of $\psi$ will enable us to compare the corresponding operator with an operator $S$ satisfying the hypotheses of Theorem 2 . After choosing $\psi$ we shall need to use a unitary equivalence of the following kind.

Suppose that $J \subseteq \mathbb{R}$ is another closed interval and that $\sigma: J \rightarrow I$ is a $C^{1}$ homeomorphism with $\sigma^{\prime}(t)>0$ on $J$, then $\sigma$ induces a unitary operator $U_{\sigma}$ mapping $L^{2}(I)$ onto $L^{2}(J)$ :

$$
U_{\sigma} f(s)=f(\sigma(s)) \sigma^{\prime}(s)^{\frac{1}{2}} \quad\left(f \in L^{2}(I), s \in J\right) .
$$

The inverse $U_{\sigma}^{-1}=U_{\sigma}^{*}: L^{2}(J) \rightarrow L^{2}(I)$ is given by an analogous formula involving $\sigma^{-1}$. If $K$ is an $L^{2}$-integral kernel on $I \times I$, then the function $\sigma K$ on $J \times J$

$$
\sigma K(s, t)=\sigma^{\prime}(s)^{\frac{1}{2}} K(\sigma(s), \sigma(t)) \sigma^{\prime}(t)^{\frac{1}{2}} \quad(s, t \in J)
$$


is an $L^{2}$-kernel on $J \times J$. A simple integration by substitution argument is enough to verify that

$$
T_{\sigma K J}=U_{\sigma} T_{K I} U_{\sigma}^{*}
$$

so that $T_{\sigma K J}$ and $T_{K I}$ have exactly the same eigenvalues.

3. Proof of Theorem 4 when $c \leq-1$. The method of Section 3 of [11] by proving Theorem 4 for the case $c>-1$ breaks down here because the function $\psi(u)=u^{c}$ is not a member of $P_{c}(0, \infty)$ : it is not locally integrable. For the case $c \leq-1$, more indirect methods will be needed, and we shall have to give separate proofs of the two propositions

$$
\begin{aligned}
n^{c} q\left(\frac{b-a}{b+a}\right)^{n} & =O\left(\lambda_{n}(T)\right), \\
\lambda_{n}(T) & =O\left(n^{c} q\left(\frac{b-a}{b+a}\right)^{n}\right) .
\end{aligned}
$$

Once we fix $c \leq-1$, Lemma 8 allows us to verify (3.1) for just one $\psi \in P_{c}(0, \infty)$, and likewise for (3.2). But it will be necessary for us to make two choices of $\psi$ : one to prove (3.1) and the other to prove (3.2).

Let us now fix $I=[a, b]$, where $0<a<b$. The Möbius transformation

$$
\sigma(s)=\frac{1+s}{1-s}
$$

maps the open interval $(-1,1)$ in a strictly increasing fashion onto the open half-line $(0, \infty)$ : its derivative $\sigma^{\prime}(s)=2 /(1-s)^{2}$ is everywhere positive. Let us define

$$
\alpha=\sigma^{-1}(a), \beta=\sigma^{-1}(b), J=[\alpha, \beta],
$$

so that $-1<\alpha<\beta<1$ and $\sigma(J)=I$. It is elementary that

$$
\sigma^{-1}(s)=\frac{s-1}{s+1}, \sigma^{-1 \prime}(s)=\frac{2}{(1+s)^{2}}
$$

and that

$$
\frac{\beta-\alpha}{1-\alpha \beta}=\frac{\sigma^{-1}(b)-\sigma^{-1}(a)}{1-\sigma^{-1}(a) \sigma^{-1}(b)}=\frac{b-a}{b+a} .
$$

The significance of (3.6) will be seen by referring to the statements of Theorems 2 and 4. Let $U=U_{\sigma}: L^{2}(I) \rightarrow L^{2}(J)$ be the unitary operator in (2.4) with $\sigma$ as in (3.3). We assume throughout that $I, J, \sigma$ and $U$ are as above.

We start our discussion of (3.1) by recalling the result, Lemma 2 of [7]. It states that if $\frac{1}{q}$ and $r$ are continuous, positive definite kernels on $J \times J$ and if

$$
|r(s, t)|<q(s, t) \quad(s, t \in J),
$$


then

$$
\frac{1}{q(s, t)-r(s, t)} \geq \geq^{\circ} \frac{1}{q(s, t)} \quad(s, t \in J) .
$$

A detailed proof is given in [7], but it is in fact elementary, because the left-hand side of (3.8) is just

$$
\sum_{n=0}^{\infty} \frac{r(s, t)^{n}}{q(s, t)^{n+1}} \geq^{\circ} \frac{1}{q(s, t)} \quad(s, t \in J),
$$

by Lemma 7(i); the compactness of $J$ and (3.7) ensure that the series is uniformly convergent on $J \times J$. It is a corollary of this result that we shall need.

LEMMA 9. Let $1 / p$, r be continuous, positive definite kernels on $J \times J$ such that

$$
2|r(s, t)|<p(s, t) \quad(s, t \in J) .
$$

If $1 /(p+r)$ and $r$ are positive definite, then

$$
\frac{1}{p(s, t)} \geq \geq^{\circ} \frac{1}{p(s, t)+r(s, t)} \quad(s, t \in J) .
$$

Proof. Put $q=p+r$.

Of course, this lemma is true for any closed interval $J$, but in the present discussion $J=[\alpha, \beta] \subseteq(-1,1)$ as in (3.4).

Put $c=-\rho$, so that $\rho>1$. We shall verify (3.1) in the case when

$$
\psi(u)=\int_{0}^{\delta} x^{\rho-1} e^{-x u} d x \quad(u \geq 0) .
$$

Here $\delta>0$ is fixed, though we shall place an upper bound on its value later on. Clearly $\psi$ is positive and continuous and, by Watson's lemma [2, Chapter 6], $\psi(u) \simeq u^{-\rho}$, so that $\psi \in P_{c}(0, \infty)$. In this case we have

$$
\begin{aligned}
K(s, t) & =\int_{0}^{\infty} \int_{0}^{\delta} x^{\rho-1} e^{-x u} d x e^{-u(s+t)} d u \\
& =\int_{0}^{\delta} x^{\rho-1} \int_{0}^{\infty} e^{-x u} e^{-u(s+t)} d u d x \\
& =\int_{0}^{\delta} \frac{x^{\rho-1}}{x+s+t} d x \quad(s, t \in I)
\end{aligned}
$$

This is the kernel of $T$. Our aim is to define an integral operator $S$ on $L^{2}(J)$ as in Theorem 2 such that $S \leq U T U^{*}$. The kernel of $U T U^{*}$ is

$$
\begin{aligned}
\sigma K(s, t) & =\sigma^{\prime}(s)^{\frac{1}{2}} K(\sigma(s), \sigma(t)) \sigma^{\prime}(t)^{\frac{1}{2}} \\
& =\int_{0}^{\delta} x^{\rho-1} \frac{2}{(1-s)(x+\sigma(s)+\sigma(t))(1-t)} d x \\
& =\int_{0}^{\delta} \frac{x^{\rho-1}}{1-s t+\frac{1}{2} x(1-s)(1-t)} d x \quad(s, t \in J),
\end{aligned}
$$


see (2.5) and (3.3). We shall construct $S$ by applying Lemma 9 to the kernels under the integral sign in (3.12).

For $x \in[0, \delta]$, let $T_{x}$ be the integral operator on $L^{2}(I)$ with kernel

$$
K_{x}(s, t)=\frac{1}{x+s+t} \quad(s, t \in I)
$$

and let $R_{x}$ be the integral operator on $L^{2}(J)$ with kernel

$$
L_{x}(s, t)=\frac{1}{1-s t+\frac{1}{2} x(1-s)(1-t)} \quad(s, t \in J) .
$$

Since the function $\phi(u)=e^{-x u}$ belongs to $P(0, \infty)$, the transition from (3.10) to (3.11) shows that each $K_{x}$ is positive definite on $I \times I$ (simply ignore the term $x^{\rho-1}$ and the $d x$ integration from 0 to $\delta$ ). Likewise, (3.12) shows that for each $x, L_{x}=\sigma K_{x}$, or equivalently $U T_{x} U^{*}=R_{x}$. So each $L_{x}$ is positive definite on $J \times J$.

We shall now apply Lemma 9 to $L_{x}$ by putting $p(s, t)=1-s t+\frac{1}{2} x(1-s)(1-t)$ and $r(s, t)=\frac{1}{2} x(1+s)(1+t)$. This last kernel has rank 1 and is positive definite: It is chosen so that $p+r$ is a polynomial in $x$ and st only. Thus, by Lemma 9,

$$
L_{x}(s, t)=\frac{1}{p(s, t)} \geq^{\circ} \frac{1}{p(s, t)+r(s, t)}=\frac{1}{(1+x)-(1-x) s t}(s, t \in J)
$$

provided

$$
x(1+s)(1+t)<1-s t+\frac{1}{2} x(1-s)(1-t),
$$

for all $s, t \in J:$ We do not need modulus signs on the left because $-1<\alpha \leq s, t \leq \beta<$ 1 , for all $s, t \in J$. For the same reason (3.15) will be true when $x$ is sufficiently small. Indeed, if we choose $\delta>0$ so that $\delta(1+\beta)^{2}<1-\beta^{2}$, then (3.15) will be true for all $s, t \in J$ and all $x \in[0, \delta]$.

All that we need to do now is integrate from 0 to this $\delta$. Thus, if $g \in L^{2}(J)$, then by (3.12), (3.13) and (3.14),

$$
\begin{aligned}
\left\langle U T U^{*} g, g\right\rangle & =\int_{J} \int_{J} \sigma K(s, t) \overline{g(s)} g(t) d t \\
& =\int_{J} \int_{J} \int_{0}^{\delta} x^{\rho-1} L_{x}(s, t) \overline{g(s)} g(t) d x d t d s \\
& =\int_{0}^{\delta} x^{\rho-1} \int_{J} \int_{J} L_{x}(s, t) \overline{g(s)} g(t) d t d s d x \\
& \geq \int_{0}^{\delta} x^{\rho-1} \int_{J} \int_{J} \overline{(1+x)-(1-x) s t} d t d s d x \\
& =\int_{J} \int_{J} L(s, t) \overline{g(s)} g(t) d t d s,
\end{aligned}
$$

where

$$
L(s, t)=\int_{0}^{\delta} \frac{x^{\rho-1}}{(1+x)-(1-x) s t} d x \quad(s, t \in J) .
$$


So if $S$ is the integral operator on $L^{2}(J)$ with kernel $L$, then

$$
U T U^{*} \geq S
$$

Now, for all $s, t \in J$,

$$
L(s, t)=\sum_{n=0}^{\infty} a_{n} s^{n} t^{n}
$$

where

$$
a_{n}=\int_{0}^{\delta} x^{\rho-1} \frac{(1-x)^{n}}{(1+x)^{n+1}} d x>0 \quad(n \geq 0) .
$$

A simple exercise on Watson's lemma shows that $a_{n} \simeq n^{-\rho}=n^{c}$ (for a detailed proof see Lemma 1 of [7]). It follows from (3.16), Theorem 2 and (3.6) that

$$
n^{c} q\left(\frac{b-a}{b+a}\right)^{n} \simeq \lambda_{n}(S) \leq \lambda_{n}\left(U T U^{*}\right)=\lambda_{n}(T),
$$

so that (3.1) is now verified.

To verify (3.2) we again put $c=-\rho$, where $\rho \geq 1$, but this time our special function is $\psi(u)=1 /(1+u)^{\rho}$. Thus,

$$
K(s, t)=\int_{0}^{\infty} \frac{e^{-u(s+t)}}{(1+u)^{\rho}} d u \quad(s, t \in I),
$$

and, for all $f \in L^{2}(I)$

$$
\begin{aligned}
\langle T f, f\rangle & =\int_{I} \int_{I} \overline{f(s)} f(t) \int_{0}^{\infty} \frac{e^{-u(s+t)}}{(1+u)^{\rho}} d u d t d s \\
& =\int_{0}^{\infty} \frac{1}{(1+u)^{\rho}} \int_{I} \int_{I} \overline{f(s)} f(t) e^{-u(s+t)} d t d s d u .
\end{aligned}
$$

Let us suppose, until further notice, that $\rho>1$ is not an integer, and let us choose and fix an integer $m$ such that $2 m>\rho$. We shall analyse the integral in (3.18), but only for $f$ in a certain closed subspace $E \subseteq L^{2}(I)$ ) of finite codimension $m$. We shall be able to construct a positive integral operator $S$ on $L^{2}(J)$ satisfying the hypotheses of Theorem 2 such that

$$
T \leq V S V^{*}+R
$$

where $V: L^{2}(J) \rightarrow L^{2}(I)$ is a continuous operator and $R: L^{2}(J) \rightarrow L^{2}(I)$ is continuous and symmetric. The operator $R$ will have finite rank $\leq 4 m$ and comprise the sum of two rank $2 m$ operators. These will be accumulated as we go, in a process reminiscent of the way in which awkward sets of points are consigned one after the other into an unimportant set of measure zero. The operator $V$ will comprise the product of three operators, and these will be much more significant.

We define the subspace $E$ by

$$
E=\left\{f \in L^{2}(I): \int_{I} f(t) t^{n} d t=0, \quad 0 \leq n<m\right\} .
$$


Thus $E$ is the orthogonal complement of the space of all polynomials of degree $<m$, so that $E$ has codimension $m$. Let us note here that if $f \in E$, then $\bar{f} \in E$, but that $|f| \notin E$ unless $f=0$.

For $f \in E$, let $L f$ denote the Laplace transform of $E$ :

$$
L f(z)=\int_{I} f(t) e^{-z t} d t \quad(z \in \mathbb{C}),
$$

then $L f$ is an entire function and, differentiating under the integral sign, we see that $L f$ has a zero at 0 of order $\geq m$. Let us also define

$$
F(z)=L f(z) L \bar{f}(z)=\int_{I} \int_{I} \overline{f(s)} f(t) e^{-z(s+t)} d t d s \quad(z \in \mathbb{C}),
$$

so that $F$ is entire and has a zero at 0 of order $\geq 2 m>\rho$.

Now if $f \in E$, then by (3.18) and (3.20) we have

$$
\begin{aligned}
\langle T f, f\rangle & =\int_{0}^{\infty} \frac{1}{(1+u)^{\rho}} F(u) d u \\
& \leq \int_{0}^{\infty} \frac{1}{u^{\rho}} F(u) d u \\
& =\int_{0}^{\infty} u^{-\rho} \int_{I} \int_{I} \overline{f(s)} f(t) e^{-u(s+t)} d t d s d u
\end{aligned}
$$

If we write the last two integrals in (3.22) as a product of integrals and apply Schwarz's inequality, we see that $F(u)=O\left(e^{-2 a u}\right)$ as $u \rightarrow \infty$. So, since $a>0$ and $F$ has a zero order $\geq 2 m$ at 0 , it follows that the integral in (3.21) is absolutely convergent, hence finite when $f \in E$. On the other hand, unless $f=0$, the repeated integral in (3.22) is definitely not absolutely convergent; in particular, we are not at liberty to interchange the order of integration in (3.22).

We can overcome this difficulty with the familiar ploy of integrating a singlevalued branch of the $d u$ integrand along a Hankel loop surrounding the half-line $[0, \infty)$ : the notation we require is made explicit as follows. For $r>0$, let $\gamma(r)$ be the negatively oriented boundary of the $r$-neighbourhood of the half-line $[0, \infty)$. When $r \rightarrow 0, \gamma_{r}$ shrinks to two descriptions of $[0, \infty)$, one in each direction. For $z \notin[0, \infty)$ we put $z^{-\rho}=|z|^{-\rho} \exp (-i \arg z)$, where $0<\arg z<2 \pi$. Thus $z \mapsto z^{-\rho}$ defines a singlevalued analytic function on $\mathbb{C}-[0, \infty)$. The limiting values of $z^{-\rho}$ along the cut $[0, \infty)$ are given by

$$
\lim _{y \downarrow 0}(x+i y)^{-\rho}=x^{-\rho}, \quad \lim _{y \uparrow 0}(x+i y)^{-\rho}=e^{-2 \pi i \rho} x^{-\rho} \quad(x>0) .
$$

What we need to know about loop integrals is summarised in the following lemma. Our non-integer constant $\rho>1$ is still fixed.

LEMMA 10. Let $G$ be an entire function, and suppose that there exist constants $M, \delta, X>0$ such that

$$
|G(z)| \leq M e^{-\delta x}, \text { for all } x=\operatorname{Re} z \geq X
$$


(i) For all $r>0$ the contour integral

$$
\int_{\gamma(r)} z^{-\rho} G(z) d z
$$

exists and is absolutely convergent with respect to arc length measure.

(ii) The value $H(r)$ of the integral in (i) is independent of the choice of $r \in(0, \infty)$.

(iii) If $\lambda>0$, then for all $r>0$,

$$
\int_{\gamma(r)} z^{-\rho} G(z) d z=\int_{\gamma(r)} \lambda^{-\rho+1} \omega^{-\rho} G(\lambda \omega) d \omega
$$

(iv) If in addition $G$ has a zero at 0 of order $\leq 2 m$, then for all $r>0$,

$$
\int_{\gamma(r)} z^{-\rho} G(z) d z=\left(1-e^{-2 \pi i \rho}\right) \int_{0}^{\infty} x^{-\rho} G(x) d x
$$

Proof. We leave the proof to the reader, but some comment is in order about (iii). If we evaluate left-hand integral using the substitution $z=\lambda \omega$, then we certainly replace the integrand with the one on the right, but, apriori, the left-hand contour $\lambda(r)$ should be replaced with its counter-image $\gamma(r / \lambda)$. But this last contour can be replaced with $\gamma(r)$, because the new entire function on the right satisfies the same kind of inequality as does $G$. This item is less trite than it might seem at first sight. In the application we have in mind, $G$ will depend on parameters $s, t \in J$. We shall start with the parametrized integral on the left taken over the same contour for every $s, t \in J$. We shall then make the parametrized substitutions $z=\lambda(s, t) \omega$, where each $\lambda(s, t)>0$. In the new integral on the right we will not need a parametrized family of contours: We can choose the same fixed contour, for all $s, t \in J$.

In the rest of this section we shall have to pay a good deal of attention to matters of rigour as well as manipulation so that the reader is not too distracted referring back to different parts of the paper. We give here a brief recapitualation of our notation.

We are given an interval $I=[a, b]$, where $a>0$. The subspace $E \subseteq L^{2}(I)$ is the orthogonal complement of the space of all polynomials of degree $<m$. Here $2 m>$ $\rho>1$ and $\rho \notin \mathbb{Z}$. The function $\sigma$, its inverse $\sigma^{-1}$ and their derivatives are given by

$$
\sigma(s)=\frac{1+s}{1-s}, \sigma^{\prime}(s)=\frac{2}{(1-s)^{2}}, \sigma^{-1}(s)=\frac{s-1}{s+1}, \sigma^{-1 \prime}(s)=\frac{2}{(1+s)^{2}} .
$$

The interval $J \subseteq(-1,1)$ is defined by

$$
J=\sigma^{-1}(I)=[\alpha, \beta]=\left[\sigma^{-1}(a), \sigma^{-1}(b)\right] .
$$

The unitary operators $U: L^{2}(I) \rightarrow L^{2}(J)$ and $U^{*}=U^{-1}: L^{2}(J) \rightarrow L^{2}(I)$ are given by

$$
\begin{aligned}
U f(s) & =f(\sigma(s)) \sigma^{\prime}(s)^{\frac{1}{2}}\left(f \in L^{2}(I), s \in J\right), U^{*} g(s) \\
& =g\left(\sigma^{-1}(s)\right) \sigma^{-1 \prime}(s)^{\frac{1}{2}}\left(g \in L^{2}(J), s \in I\right)
\end{aligned}
$$


Given $f \in E$, the entire function $F$ is defined by

$$
F(z)=\int_{I} \int_{I} \overline{f(s)} f(t) e^{-z(s+t)} d t d s=\left(\int_{I} \overline{f(s)} e^{-z s} d s\right)\left(\int_{I} f(t) e^{-z t} d t\right) \quad(z \in \mathbb{C})
$$

it as a zero order $\geq 2 m$ at 0 .

Now let $f$ be a fixed but arbitrary element of $E$; then, by Schwarz's inequality,

$$
|F(z)| \leq\|f\|^{2}\left(\frac{e^{-2 a x}-e^{-2 b x}}{2 x}\right)(z \in \mathbb{C}) ;
$$

so by (3.21), (3.22) and Lemma 10 (iv),

$$
\begin{aligned}
\langle T f, f\rangle & \leq\left(1-e^{-2 \pi i \rho}\right)^{-1} \int_{\gamma(r)} z^{-\rho} F(z) d z \\
& =\left(1-e^{-2 \pi i \rho}\right)^{-1} \int_{\gamma(r)} z^{-\rho} d z\left(\int_{I} \overline{f(s)} e^{-z s} d s\right)\left(\int_{I} f(t) e^{-z t} d t\right),
\end{aligned}
$$

for any $r>0$. Another application of Schwarz's inequality, to the inner integrals, shows us that the repeated integral in (3.23) is absolutely convergent, unlike the one in (3.22). It will now be convenient to write $f=U^{*} g$, where $g \in L^{2}(J)$. Thus

$$
\begin{aligned}
\langle T f, f\rangle \leq & \left(1-e^{-2 \pi i \rho}\right)^{-1} \int_{\gamma(r)} z^{-\rho} \int_{I} \int_{I} \overline{g\left(\sigma^{-1}(s)\right)} g\left(\sigma^{-1}(t)\right) \sigma^{-1 \prime}(s)^{\frac{1}{2}} \sigma^{-1 \prime} \\
& (t)^{\frac{1}{2}} e^{-z(s+t)} d t d s d z .
\end{aligned}
$$

We now integrate by substitution in the inner integrals, replacing $s$ with $\sigma(s)$ and $t$ with $\sigma(t)$ : we obtain

$$
\begin{aligned}
\langle T f, f\rangle & \leq\left(1-e^{2 \pi i \rho}\right)^{-1} \int_{\gamma(r)} z^{-\rho} \int_{J} \int_{J} \frac{2 \overline{g(s)} g(t)}{(1-s)(1-t)} \exp (-z(\sigma(s)+\sigma(t))) d t d s d z \\
& =\left(1-e^{2 \pi i \rho}\right)^{-1} \int_{\gamma(r)} z^{-\rho} \int_{J} \int_{J} \frac{2 \overline{g(s)} g(t)}{(1-s)(1-t)} \exp \left(\frac{2 z(s t-1)}{(1-s)(1-t)}\right) d t d s d z .
\end{aligned}
$$

Necessarily this last repeated integral is still absolutely convergent; so we can interchange the order of integration, thus

$$
\langle T f, f\rangle \leq\left(1-e^{-2 \pi i \rho}\right)^{-1} \int_{J} \int_{J} \frac{2 \overline{g(s)} g(t)}{(1-s)(1-t)} \int_{\gamma(r)} z^{-\rho} \exp \left(\frac{2 z(s t-1)}{(1-s)(1-t)}\right) d z d t d s
$$

Since the $d z$ integral is now innermost we can evaluate it by substitution. For each fixed $s, t \in J$ we shall replace $z$ with $\frac{1}{2}(1-s)(1-t) z$, so that the argument of the exponential function becomes $z(s t-1)$. Clearly $\frac{1}{2}(1-s)(1-t)>0$ if $s, t \in J$. Moreover, if

$$
G(s, t, z)=\exp \left(\frac{2 z(s t-1)}{(1-s)(1-t)}\right)(z \in \mathbb{C}, s, t \in J),
$$


then $G$ satisfies an inequality of the type given in Lemma 10; this is because $(s t-1) /(1-s)(1-t)<0$ on $J \times J$. So, by Lemma 10 (i), (ii) and (iii),

$$
\langle T f, f\rangle \leq \int_{J} \int_{J} \frac{2^{\rho+1} \overline{g(s)} g(t)}{(1-s)^{\rho+1}(1-t)^{\rho+1}}\left(1-e^{-2 \pi i \rho}\right)^{-1} \int_{\gamma(r)} z^{-\rho} e^{-z} e^{z s t} d z d t d s .
$$

This is true for all $r>0$ (independent of $s, t$ ).

We can now interpret the right-hand side of (3.24) in terms of operators. Let $M$ be the multiplication operator on $L^{2}(J)$ :

$$
M h(s)=\frac{2^{\frac{1}{2}(\rho+1)}}{(1-s)^{\rho+1}} h(s) \quad\left(h \in L^{2}(J), s \in J\right)
$$

so that $M$ is continuous and symmetric and

$$
\|M\|=\sup _{s \in J}\left|\frac{2^{\frac{1}{2}(\rho+1)}}{(1-s)^{\rho+1}}\right|=\frac{2^{\frac{1}{2}(\rho+1)}}{(1-\beta)^{\rho+1}} .
$$

Let $S^{\prime}$ be the integral operator on $L^{2}(J)$ with kernel

$$
L^{\prime}(s, t)=\left(1-e^{-2 \pi i \rho}\right)^{-1} \int_{\gamma(r)} z^{-\rho} e^{-z} e^{z s t} d z \quad(s, t \in J),
$$

then

$$
\begin{aligned}
L^{\prime}(s, t) & =\left(1-e^{-2 \pi i \rho}\right)^{-1} \int_{\gamma(r)} \sum_{n=0}^{\infty} \frac{s^{n} t^{n}}{n !} z^{-\rho+n} e^{-z} d z \quad(s, t \in J) \\
& =\sum_{n=0}^{\infty} \frac{(n-\rho) !}{n !} s^{n} t^{n},
\end{aligned}
$$

absolutely and uniformly on $J \times J$. The coefficients in (3.27) are real, so $S^{\prime}$ is symmetric. We can now rewrite (3.24) as

$$
\langle T f, f\rangle \leq\left\langle S^{\prime} M g, M g\right\rangle=\left\langle M S^{\prime} M g, g\right\rangle .
$$

Since $2 m>\rho$, the coefficients in (3.27) are positive for $n \geq 2 m$, and asymptotic to $n^{-\rho}$, by Stirling's formula; but the first few coefficients alternate in sign. With this problem in mind let us define

$$
L(s, t)=\sum_{n=0}^{2 m-1} s^{n} t^{n}+\sum_{n=2 m}^{\infty} \frac{(n-\rho) !}{n !} s^{n} t^{n}, \quad(s, t \in J),
$$

and define $S$ to be the positive integral operator on $L^{2}(J)$ with kernel $L$; clearly $S$ satisfies the hypotheses of Theorem 2 with $c=-\rho$. From (3.28) we now have

$$
\langle T f, f\rangle \leq\langle M S M g, g\rangle+\left\langle M\left(S^{\prime}-S\right) M g, g\right\rangle \text {. }
$$


Since the kernel $L^{\prime}-L$ of $S^{\prime}-S$ is a real polynomial in $s t$ of degree $2 m-1$, we see that $M\left(S^{\prime}-S\right) M$ has rank $2 m$. Replacing $g$ with $U f$ we see that for all $f \in E$,

$$
\langle T f, f\rangle \leq\left\langle U^{*} M S M U f, f\right\rangle+\left\langle R_{1} f, f\right\rangle,
$$

where $R_{1}=U^{*} M\left(S^{\prime}-S\right) M U$ : a continuous symmetric operator on $L^{2}(I)$ of rank $2 m$.

Let $P$ be the orthogonal projection of $L^{2}(I)$ onto $E$. The null-space of $P$ is the space of all polynomials of degree $<m$, so the rank of $R_{2}=T-P T P$ is $\leq 2 m$. Now, if $f$ is any element of $L^{2}(I)$, then from (3.30), we have

$$
\begin{aligned}
\langle T f, f\rangle & =\langle P T P f, f\rangle+\left\langle R_{2} f, f\right\rangle \\
& \leq\left\langle P U^{*} M S U M P f, f\right\rangle+\left\langle U^{*} R_{1} U f, f\right\rangle+\left\langle R_{2} f, f\right\rangle .
\end{aligned}
$$

Thus

$$
T \leq V S V^{*}+R,
$$

where

$$
V=P U^{*} M, \quad R=U^{*} R_{1} U+R_{2} .
$$

$V: L^{2}(J) \rightarrow L^{2}(I)$ is continuous and $R$ is a continuous symmetric operator on $L^{2}(I)$ of rank $\leq 4 m$. It follows from Lemma 6 (ii) and (iv) that

$$
\lambda_{n}(T) \leq\|V\|^{2} \lambda_{n-4 m}(S) \quad(n \geq 4 m) .
$$

Now, by Theorem 2 and (3.6),

$$
\lambda_{n}(S) \simeq n^{-\rho} q\left(\frac{\beta-\alpha}{1-\alpha \beta}\right)^{n}=n^{-\rho} q\left(\frac{b-a}{b+a}\right)^{n},
$$

so that $\lambda_{n-4 m}(S)=O\left(\lambda_{n}(S)\right)$ and hence by (3.31),

$$
\lambda_{n}(T)=O\left(n^{-\rho} q\left(\frac{b-a}{b+a}\right)^{n}\right) .
$$

This establishes (3.2), so the proof of Theorem 4 is now complete in the case where $c<-1$ is not an integer.

Finally, let us assume that $c=-N$, where $N \geq 1$ is an integer. Relation (3.1) has already been verified, so it only remains for us to establish (3.2). We shall do this by letting $\rho$ decrease to $N$ in (3.32). If $\rho$ is to be variable we must take account of this fact in our notation. Rather than use the special functions $\psi(u)=(1+u)^{-\rho}$ to label our operators and kernels, as we did in Definition 3, it is simpler to label them with $\rho$. In (3.32) the integer $m$ was chosen to satisfy $2 m>\rho$. We must ensure that $m$ does not vary with $\rho$ as we take the limit. This can easily be done by restricting $N<\rho<N+1$ throughout and insisting that $m$ is fixed and satisfies $2 m \geq N+1$.

Now, for $N \leq \rho<N+1$, let

$$
K_{\rho}(s, t)=\int_{0}^{\infty} \frac{e^{-u(s+t)}}{(1+u)^{\rho}} d u \quad(s, t \in J),
$$


(see (3.17)) and $T_{\rho}$ be the integral operator on $L^{2}(I)$ with kernel $K_{\rho}$. Let $S_{\rho}$ be the integral operator on $L^{2}(J)$ with kernel

$$
L_{\rho}(s, t)=\sum_{n=0}^{2 m-1} s^{m} t^{n}+\sum_{n=2 m}^{\infty} \frac{(n-\rho) !}{n !} s^{n} t^{n} \quad(s, t \in J),
$$

(see (3.29)). We define $V_{\rho}=P U^{*} M_{\rho}$ (see (3.31)); here $P$ is a fixed projection on $L^{2}(I)$, $U^{*}: L^{2}(J) \rightarrow L^{2}(I)$ is a fixed unitary operator, and $M_{\rho}$ is the multiplication operator

$$
M_{\rho} h(s)=\frac{2^{\frac{1}{2}(\rho+1)}}{(1-s)^{\rho+1}} h(s) \quad\left(h \in L^{2}(J), s \in J\right),
$$

as in (3.25). We can now rewrite (3.32) as

$$
\lambda_{n}\left(T_{\rho}\right) \leq\left\|V_{\rho}\right\|^{2} \lambda_{n-4 m}\left(S_{\rho}\right) \quad(n \geq 4 m, N<\rho<N+1)
$$

and because $\left\|U^{*}\right\|=\|P\|=1$, (3.26) shows that

$$
\lambda_{n}\left(T_{\rho}\right) \leq \frac{2^{\rho+1}}{(1-\beta)^{2 \rho+2}} \lambda_{n-4 m}\left(S_{\rho}\right) \quad(n \geq 4 m, N<\rho<N+1) .
$$

Now suppose that $\rho$ decreases to $N$. It is easy to verify that $K_{\rho} \rightarrow K_{N}$ uniformly on $I \times I$ and that $L_{\rho} \rightarrow L$ uniformly on $J \times J$, so that $\left\|K_{\rho}-K_{N}\right\| \rightarrow 0$ and $\left\|L_{\rho}-L_{N}\right\| \rightarrow 0$. So by (3.35) and Lemma 6 (i),

$$
\lambda_{n}\left(T_{N}\right) \leq \frac{2^{N+1}}{(1-\beta)^{2 N+2}} \lambda_{n-4 m}\left(S_{N}\right) \quad(n \geq 4 m) .
$$

Once again we have $\lambda_{n-4 m}\left(S_{N}\right)=O\left(\lambda_{n}\left(S_{N}\right)\right)$, so that

$$
\lambda_{n}\left(T_{N}\right)=O\left(\lambda_{n}\left(S_{N}\right)\right)=O\left(n^{-N} q\left(\frac{b-a}{b+a}\right)^{n}\right) .
$$

This establishes (3.2) for $c=-N$ and the proof of Theorem 4 is now complete.

\section{REFERENCES}

1. M. Abramowitz and I. A. Stegun, Handbook of mathematical functions (Dover Publications, New York, 1965).

2. E. T. Copson, Asymptotic expansions (Cambridge University Press, New York, 1965).

3. A. G. Greenhill, The applications of elliptic functions (Dover Publications, New York, 1959) (Kessinger Publishing, Montana, 2008).

4. G. Little and J. B. Reade, Eigenvalues of analytic kernels, SIAM J. Math. Anal. 15 (1984), 133-136.

5. G. Little, Asymptotic estimates of the eigenvalues of certain positive Fredholm operators II. Proc. Camb. Philos. Soc. 101 (1987), 535-545.

6. G. Little, Equivalences of positive integral operators with rational kernels, Proc. London Math. Soc. 62 (1991), 403-426.

7. G. Little, Eigenvalues of positive power series kernels, Bull. London Math. Soc. 28 (1996), 43-50.

8. E. H. Neville, Jacobian elliptic functions (Oxford University Press, London, 1951). 
9. E. G. Phillips, Some topics in complex analysis (Pergamon Press, Oxford, 1966).

10. F. Riesz and B. Sz-Nagy, translated by L. F. Boron, Functional analysis (Frederick Ungar Publishing Co., New York, 1955).

11. Y. Soykan, Asymptotic behaviour of eigenvalues of certain positive integral operators, Glasgow Math. J. 51 (2009), 149-159.

12. H. Widom, Asymptotic behaviour of the eigenvalues of certain integral equations, Arch. Rational Mech. Anal. 17 (1964), 215-229. 